\title{
BOLD Repetition Decreases in Object-Responsive Ventral Visual Areas Depend on Spatial Attention
}

E. Eger, R.N.A. Henson, J. Driver and R. J. Dolan

$J N$ 92:1241-1247, 2004. First published Mar 31, 2004; doi:10.1152/jn.00206.2004

You might find this additional information useful...

This article cites 21 articles, 3 of which you can access free at:

http://jn.physiology.org/cgi/content/full/92/2/1241\#BIBL

This article has been cited by 5 other HighWire hosted articles:

Orientation-Selective Adaptation to First- and Second-Order Patterns in Human Visual Cortex

J. Larsson, M. S. Landy and D. J. Heeger

J Neurophysiol, February 1, 2006; 95 (2): 862-881.

[Abstract] [Full Text] [PDF]

Object-Selective Cortex Exhibits Performance-Independent Repetition Suppression

R. Sayres and K. Grill-Spector

J Neurophysiol, February 1, 2006; 95 (2): 995-1007.

[Abstract] [Full Text] [PDF]

Selective Attention Modulates Neural Substrates of Repetition Priming and "Implicit"

Visual Memory: Suppressions and Enhancements Revealed by fMRI

P. Vuilleumier, S. Schwartz, S. Duhoux, R. J. Dolan and J. Driver

J. Cogn. Neurosci., August 1, 2005; 17 (8): 1245-1260.

[Abstract] [Full Text] [PDF]

Using Functional Magnetic Resonance Imaging to Assess Adaptation and Size Invariance of Shape Processing by Humans and Monkeys

H. Sawamura, S. Georgieva, R. Vogels, W. Vanduffel and G. A. Orban

J. Neurosci., April 27, 2005; 25 (17): 4294-4306.

[Abstract] [Full Text] [PDF]

Attentional Modulation of Learning-Related Repetition Attenuation Effects in Human

Parahippocampal Cortex

D.-J. Yi and M. M. Chun

J. Neurosci., April 6, 2005; 25 (14): 3593-3600.

[Abstract] [Full Text] [PDF]

Updated information and services including high-resolution figures, can be found at:

http://jn.physiology.org/cgi/content/full/92/2/1241

Additional material and information about Journal of Neurophysiology can be found at:

http://www.the-aps.org/publications/jn

This information is current as of February 15, 2006.

Journal of Neurophysiology publishes original articles on the function of the nervous system. It is published 12 times a year (monthly) by the American Physiological Society, 9650 Rockville Pike, Bethesda MD 20814-3991. Copyright (C) 2005 by the American Physiological Society. ISSN: 0022-3077, ESSN: 1522-1598. Visit our website at http://www.the-aps.org/. 


\title{
BOLD Repetition Decreases in Object-Responsive Ventral Visual Areas
}

\section{Depend on Spatial Attention}

\author{
E. Eger, ${ }^{1,2}$ R.N.A. Henson, ${ }^{1,2}$ J. Driver, ${ }^{1,2}$ and R. J. Dolan ${ }^{1}$ \\ ${ }^{1}$ Wellcome Department of Imaging Neuroscience, London WC1N 3BG; and ${ }^{2}$ Institute of Cognitive Neuroscience, London WC1N 3AR, \\ United Kingdom
}

Submitted 2 March 2004; accepted in final form 23 March 2004

Eger, E., R.N.A. Henson, J. Driver, and R. J. Dolan. BOLD repetition decreases in object-responsive ventral visual areas depend on spatial attention. J Neurophysiol 92: 1241-1247, 2004. First published March 31, 2004; 10.1152/jn.00206.2004. Functional imaging studies of priming-related repetition phenomena have become widely used to study neural object representation. Although blood oxygenation level-dependent (BOLD) repetition decreases can sometimes be observed without awareness of repetition, any role for spatial attention in BOLD repetition effects remains largely unknown. We used fMRI in 13 healthy subjects to test whether BOLD repetition decreases for repeated objects in ventral visual cortices depend on allocation of spatial attention to the prime. Subjects performed a size-judgment task on a probe object that had been attended or ignored in a preceding prime display of 2 lateralized objects. Reaction times showed faster responses when the probe was the same object as the attended prime, independent of the view tested (identical vs. mirror image). No behavioral effect was evident from unattended primes. BOLD repetition decreases for attended primes were found in lateral occipital and fusiform regions bilaterally, which generalized across identical and mirror-image repeats. No repetition decreases were observed for ignored primes. Our results suggest a critical role for attention in achieving visual representations of objects that lead to both BOLD signal decreases and behavioral priming on repeated presentation.

\section{N T R O D U C T I O N}

Studies of visual object processing with functional magnetic resonance imaging (fMRI) have increasingly used blood oxygenation level-dependent (BOLD) repetition-suppression effects, during adaptation or "priming" paradigms, as a tool beyond basic subtraction designs for studying the neural representation of visual stimuli (e.g., see Grill-Spector et al. 1999; Henson 2003; Naccache and Dehaene 2001). Such repetition effects can provide information about the level of representation achieved in a given cortical region, by determining whether these effects generalize across specific transformations in the repeated stimulus, such as size and viewpoint in the case of object processing (Grill-Spector et al. 1999; James et al. 2002; Vuilleumier et al. 2002). fMRI studies using this approach have some parallels with the literature on behavioral priming effects [measured, e.g., with reaction times (RTs)] for objects repeated across various transformations (e.g., see Biederman and Kalocsai 1997; Bulthoff et al. 1995). It has been suggested that BOLD repetition decreases might provide a neural substrate for behavioral priming effects (Wiggs and

Address for reprint requests and other correspondence: E. Eger, Institute of Cognitive Neuroscience, 17 Queen Square, London WC1N 3AR, UK (E-mail: e.eger@fil.ion.ucl.ac.uk).
Martin 1998), although caution should be exercised in assuming a direct relationship (Henson and Rugg 2003).

A question often addressed in behavioral priming studies, but rarely in fMRI work to date, is the extent to which specific repetition effects depend on attention. Some behavioral studies show that, although explicit awareness of object identity may depend on attention, some priming effects may nevertheless be found from unattended objects, at least in some circumstances (e.g., deSchepper and Treisman 1996; Tipper 1985). However, behavioral work addressing this issue has led to variable outcomes. In some studies, "positive" priming (i.e., shorter RTs) was observed from putatively unattended objects (e.g., Stankiewicz et al. 1998), whereas others reported "negative" priming (longer RTs) when a previously unattended object was subsequently attended (e.g., Tipper 1985; Tipper and Driver 1988). In other instances, no behavioral priming was observed in the absence of attention (e.g., Crabb and Dark 1999). Researchers using purely behavioral measures have begun to suspect that the variable behavioral priming observed might reflect the combined expression of several different types of neuronal "priming" effects.

There has been surprisingly little fMRI work to date on how BOLD repetition effects for visual objects depend on attention, although in principle this might disentangle different types of priming effects that could become superimposed in purely behavioral measures. Accordingly, we used event-related fMRI here to examine the influence of spatial attention on object priming as tested by BOLD repetition decreases. Our design included repetition of the identical object image, but also repetition across a mirror reversal that substantially changed the retinal image. Our main finding is that whereas fMRI repetition-suppression effects were reliably found from an attended object for a subsequent probe (in both original and mirror conditions), these effects were eliminated for unattended objects. This demonstrates a strong dependency of BOLD repetition suppression on spatial attention to the object at initial exposure.

\section{METHODS}

\section{Subjects and imaging}

Thirteen healthy right-handed volunteers $(7$ male/ 6 female, mean age $27.7 \pm 5.8 \mathrm{yr}$ ) had normal or corrected vision. The study was approved by the Joint Ethics Committee of the National Hospital and Institute of Neurology, London. Functional images were acquired on

The costs of publication of this article were defrayed in part by the payment of page charges. The article must therefore be hereby marked "advertisement" in accordance with 18 U.S.C. Section 1734 solely to indicate this fact. 
a 3-Tesla MR system with standard head coil (Siemens Allegra, Erlangen, Germany) as T2*-weighted echoplanar image (EPI) volumes every $2 \mathrm{~s}$ (TE $30 \mathrm{~ms}, 32$ transversal slices with $30^{\circ}$ anteriorposterior angulation, voxel size $3 \times 3 \times 2 \mathrm{~mm}$, distance factor $75 \%$, approximate slice position in Fig. 2).

\section{Stimuli}

A total of 120 digitized photographs or realistic shaded renderings of asymmetrical familiar objects served as stimuli. These came from different sources (Object Databank: http://www.cog.brown.edu/ $\sim$ tarr/; MasterClips image collection: http://www.imsisoft.com; and Hemera Photo Clipart: http://www.hemera.com). Images were converted to grayscale on a white background. Object categories were household items (31), animals (18), tools (13), vehicles (12), musical instruments (6), food (6), and others (34). Stimuli were divided into 6 sets of 20 objects each, approximately equated for category content. They were rotated around the 6 experimental conditions across subjects for counterbalancing. An additional 120 objects of comparable categories served as "fillers" (see following text) in prime trials. During a "localizer" scan to determine areas responding visually to the objects, the objects from the priming study were presented in addition to "noise" versions of these stimuli (created by adding Gaussian white noise to the Fourier phase angles).

\section{Experimental protocol and task}

Stimuli were back-projected onto a translucent screen located approximately $60 \mathrm{~cm}$ above the subjects' head and viewed by a mirror on the head coil. Objects subtended approximately $4.5^{\circ}$.

In 3 sessions of about 12 min each, trials started with a red cueing square of $5^{\circ}$ for $100 \mathrm{~ms}$, centered at an eccentricity of $5.5^{\circ}$, unpredictably to left or right of central fixation (Fig. 1). This was followed by presentation of 2 concurrent objects for $100 \mathrm{~ms}$, one inside the square, the other on the opposite side of fixation (cue plus display duration was thus $200 \mathrm{~ms}$, which should be too brief for deliberate saccades to the cued object while it was still displayed ${ }^{1}$ ). Subjects were instructed to attend covertly to the object in the cued location and perform a speeded size-judgment task (press one of 2 buttons depending on whether in real life the object would fit into a shoebox). Subjects were instructed to ignore the object presented on the uncued side. After an interstimulus interval of $3 \mathrm{~s}$, a single probe object appeared centrally for $200 \mathrm{~ms}$ and subjects again performed the size judgment. The probe was either a new object, the same as the attended (or unattended) object in the preceding prime display, or a mirrorreversed version of this. There were 6 experimental conditions: primed attended; primed unattended; primed mirror image attended; primed mirror image unattended; unprimed (baseline for attended); unprimed (baseline for unattended). The 2 baselines accounted for the fact that when the attended object primed the subsequent probe, there was a congruent size-judgment response to prime and target (as in the attended baseline also), whereas when the unattended image primed the subsequent probe, responses to prime and target were incongruent, as in the unattended baseline (see Fig. 1). All experimental conditions where randomly intermingled with an intertrial interval of $3 \mathrm{~s}$. Filler objects (occupying the opposite side to the potentially probe-related object in prime displays) were randomly assigned to each trial.

Our analysis focused on immediate repetition effects (within each trial pair). Twenty different objects were used for each of the 6 experimental conditions. The trials for each object were presented twice within one session and therefore 6 times altogether. Any

\footnotetext{
${ }^{1}$ Eye position was monitored during scanning in 7 of the subjects tested with an infrared system (Model 504; Applied Sciences Laboratories, Waltham, MA). Analysis of these eye-position data showed that even for those trials selected because a saccade did occur (mean 32\% across subjects), these shifts in eye position arose only after the prime display, and thus did not change visual input from the primes themselves.
}

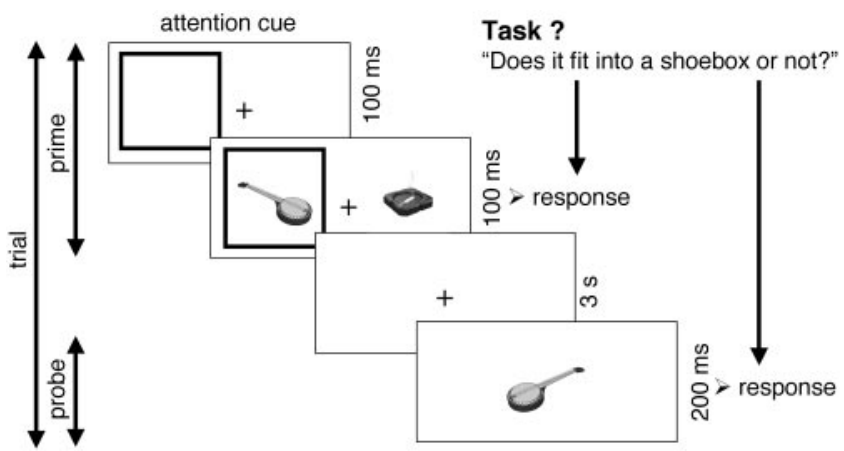

Size 4.5 deg VA, excentricity 5.5 deg VA

FIG. 1. Schematic overview of experimental trials: an attentional precue appeared for $100 \mathrm{~ms}$, followed by 2 objects for another $100 \mathrm{~ms}$, one of them in the cueing square, the other one on the opposite side of the screen. After a stimulus onset asynchrony (SOA) of $3 \mathrm{~s}$, a third object appeared in the center of the screen. During both prime and probe phase, subjects performed a size-judgment task (for the cued object in case of the prime display). Probe could be the attended or unattended picture from the prime display in original or mirror-reversed format (as here), or a new (unprimed) object with the same or different response as the preceding prime.

additional long-lag between-trial repetition effect that might in principle have occurred within a condition should apply equally to all experimental conditions and is thus unlikely to systematically confound our comparisons; session was nevertheless considered as a factor in some of our analyses.

After the main experiment, an 8-min "localizer" session mapped regions responding to the visual objects (vs. noise patterns). Subjects passively viewed 10 blocks of 12 object pictures, alternating with 10 blocks of 12 phase randomized "noise" patterns, all stimuli being presented for $500 \mathrm{~ms}$ with a stimulus onset asynchrony (SOA) of $1 \mathrm{~s}$ and blocks separated by baseline periods of $12 \mathrm{~s}$.

Stimulus presentation and recording of behavioral responses used in-house software (Cogent 2000v1.24: http://www.vislab.ucl.ac.uk/ Cogent) within Matlab6.

\section{Image processing and data analysis}

Analysis of imaging data used SPM2 (http://www.fil.ion.ucl.ac.uk/ spm2.html). Image preprocessing included realignment and unwarping, slice-time correction with middle slice as reference, spatial normalization (EPI-template), and spatial smoothing (10 mm full width at half-maximum Gaussian kernel). Responses to probe displays for the 6 experimental conditions, and separately to the 2 types of prime display for cue left or cue right, were modeled by delta functions convolved with a canonical hemodynamic response function (HRF) and its temporal derivative. Incorrect responses to probes constituted a separate regressor. Parameter estimates for all regressors were obtained by maximum-likelihood estimation, while using a temporal high-pass filter (cutoff $128 \mathrm{~s}$ ), and modeling temporal autocorrelation as an $\mathrm{AR}(1)$ process. All statistical comparisons were performed as random-effects group analyses across the 13 subjects, using one-sample $t$-tests on images of the contrasts of HRF parameter estimates.

We report activations significant at $P<0.001$, uncorrected, within the mask of object-responsive areas (see Fig. $2 A$ ), where objectresponsive areas were defined by the group contrast of objects $>$ phase-randomized noise at $P<0.001$, uncorrected, from the localizer scans. In an additional region-of-interest (ROI) analysis, parameter estimates were extracted and averaged across voxels from spherical regions of interest of 10-mm radius, centered on left and right lateral occipital (LO) and fusiform response maxima, defined individually from each subject's localizer contrast (objects $>$ noise). 

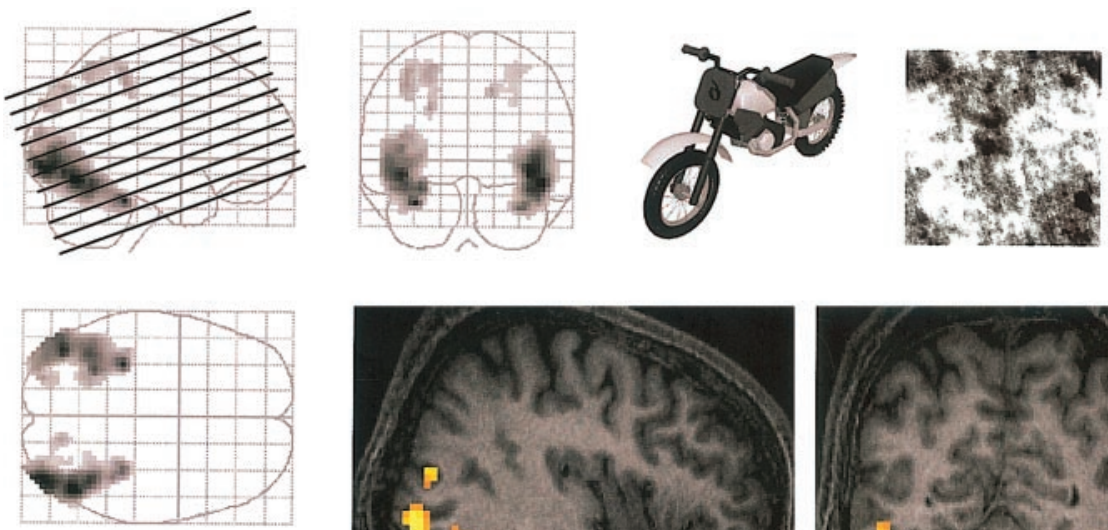

A
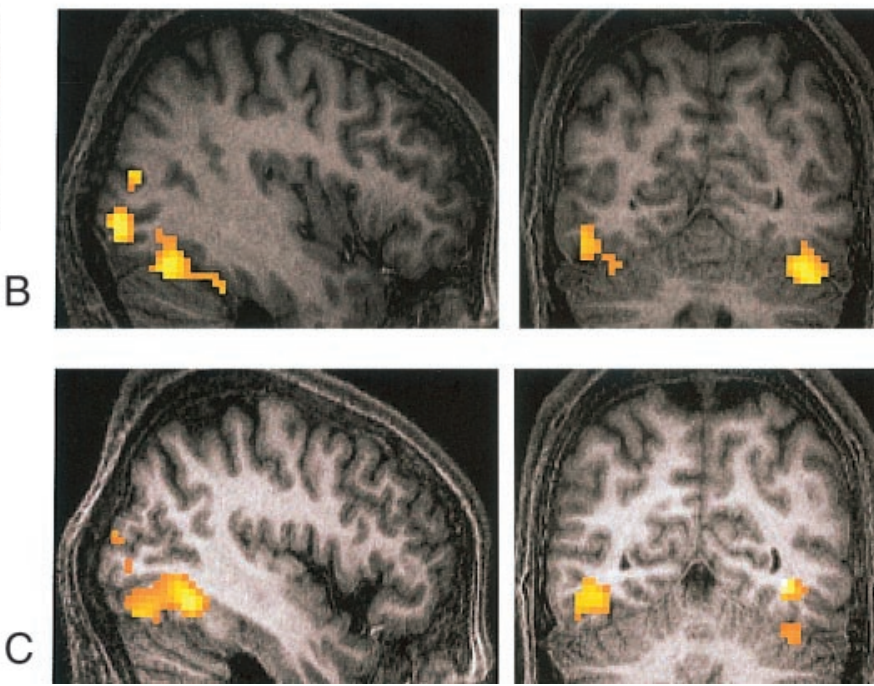

FIG. 2. Imaging findings from group analyses. $A$ : mask of object responsive regions used for smallvolume correction, which was derived from the contrast of objects $>$ phase-randomized noise, at $P>0.001$, uncorrected. Tilted lines indicate the approximate slice position during scanning. $B$ : regions expressing significant repetition decreases for attended original images. $C$ : attended mirror images, in contrast with the unprimed baseline condition. For display purposes, findings were color-coded at $P<0.005$, uncorrected (masked inclusively by localizer at $P<0.001$ ) and superimposed on the normalized structural scan of one participating subject. In both the original and mirror-image conditions, similar repetition decreases were found in lateral occipital and fusiform regions bilaterally.

\section{R E S UL T S}

\section{Behavior}

Table 1 gives mean probe RTs and percentage correct in the size-judgment task for the 6 conditions during scanning, showing shorter reaction times for conditions with attended related primes. RTs for primed conditions were subtracted from their respective baseline and analyzed in a $2 \times 2$ ANOVA (original/mirror $\times$ attended/unattended). This yielded a significant main effect of attention on priming $[F(1,12)=35.5, P<0.0001]$. The main effect of view $[F(1,12)=0.003]$ and the interaction $[F(1,12)=3.7]$ were nonsignificant, indicating equivalent priming for repetition of the same image and for mirror images, but a critical dependency on attention. Mean priming was $98 \mathrm{~ms}$ from attended objects $[t(12)=7.2, P<0.001]$ but only $5 \mathrm{~ms}$ from unattended objects $[t(12)=0.6$, n.s. $]$. Analysis of error rates yielded no significant effects, but confirmed that the priming effects in RTs for attended object were not due to speed/accuracy trade-offs. Figure $4 A$ shows individual RT priming effects for all 13 subjects, with $95 \%$ confidence intervals, for the attended and unattended conditions, demonstrating that the vast majority of subjects individually showed priming from attended objects, but not from unattended (confidence intervals for latter overlapping with zero). Thus the absence of priming from unattended objects was not caused by some subjects showing strong positive priming and others showing strong negative priming; instead, the group results are representative of most individuals.

\section{Imaging}

We were specifically interested in BOLD repetition-suppression effects in brain regions showing significant responses to objects (as defined by the localizer, which activated lateral occipital and ventral occipitotemporal regions bilaterally; see Fig. 2). To restrict further interrogation of the data to these regions, we masked and small-volume-corrected all further comparisons using the localizer contrast at $P<0.001$, uncorrected.

Repetition decreases in the event-related BOLD response as a function of object repetition were first investigated by the simple effects for each of the primed conditions subtracted from its respective baseline condition. Analyses for primed attended original and mirror images each yielded a similar pattern of repetition decrease in lateral occipital and fusiform regions bilaterally (Fig. 2, Table 2). By contrast, the same comparison for primed unattended original images and for unattended mirror images showed no effects, not even at the lower threshold of $P<0.01$, uncorrected. To directly compare repetition effects from attended and unat-

TABLE 1. Behavioral data (mean of 13 subjects $\pm S E$ )

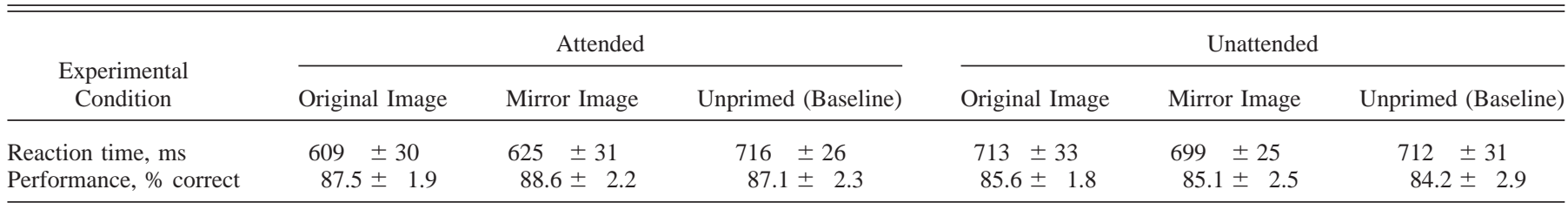


TABLE 2. Repetition decreases for priming from attended objects (separately for original and mirror images)

\begin{tabular}{clrrrl}
\hline \hline Contrast & \multicolumn{1}{c}{ Region } & \multicolumn{2}{c}{ MNI Coordinates } & \multicolumn{2}{c}{$Z$} \\
\hline $\begin{array}{c}\text { Repetition decreases } \\
\text { Attended original } \\
\text { image }\end{array}$ & Lateral occipital & 39 & -87 & -6 & 3.88 \\
& and fusiform R & 45 & -66 & -21 & 3.58 \\
& & 42 & -81 & 15 & 3.23 \\
& Lateral occipital & -36 & -90 & 12 & $4.30^{*}$ \\
& and fusiform L & -42 & -72 & -21 & 3.61 \\
& & -30 & -45 & -24 & 3.37 \\
Attended mirror & Posterior IPS L & -30 & -90 & 27 & 3.04 \\
image & Posterior to & -24 & -57 & 45 & 3.01 \\
& midfusiform R & 36 & -60 & -3 & $4.38^{*}$ \\
& & 48 & -72 & -24 & 3.37 \\
& Lateral occipital & -42 & -54 & -21 & 2.85 \\
& and fusiform L & -36 & -33 & -27 & 3.94 \\
& & -42 & -87 & 15 & 3.44 \\
& Lateral occipital R & 30 & -93 & -9 & 3.87 \\
& & 45 & -78 & 9 & 3.75 \\
& IPS L & -27 & -78 & 30 & 3.01 \\
\hline
\end{tabular}

Regions that survived correction for multiple comparisons at voxel level for the volume of areas defined by the localizer at $P<0.05$ are indicated with $*$. Other regions are reported at a threshold of $P<0.001$, uncorrected, in both priming contrast and the localizer mask.

tended objects, we further investigated these according to a $2 \times 2$ factorial design (original/mirror image $\times$ attended/unattended) after subtraction from the respective baseline conditions, analogous to the analysis of the behavioral data. The main effect of attention (repetition decrease for attended $>$ unattended) gave significant results in lateral occipital and posterior to midfusiform regions bilaterally (Fig. 3, Table 3), and thus confirmed the pattern observed in the simple-effect comparisons. No voxels showed stronger repetition decreases for unattended than attended images.

To assess effects of view, we first subtracted the primed original from primed mirror-image conditions. This comparison yielded no effects at $P<0.001$, uncorrected, nor did the reverse comparison (i.e., primed mirror image $>$ primed original image ${ }^{2}$ ).

Finally, we performed ROI analyses on lateral occipital and fusiform maxima as defined individually by each subject's localizer scan. Unlike the group SPM approach, this analysis can take into account any variability in the location of objectresponsive regions across subjects, and could potentially be more sensitive to small levels of signal change that are coherent across these individually defined regions, as it aggregated over multiple voxels in those regions (see METHODS).

Data from the 4 ROIs for each subject are plotted in Fig. $4 B$ in addition to the individual behavioral priming effects ${ }^{3}$. The ROI fMRI analysis confirmed a main effect of attention on BOLD repetition suppression $[F(1,12)=25.09, P=0.0001]$, whereas there was no difference across the regions tested, as indicated by nonsignificant interactions with region $[F(1,12)=$ $0.044]$ or laterality $[F(1,12)=0.005]$. Furthermore, the attentional influence on repetition effects did not interact with experimental session $[F(1.9,22.8)=0.284]$. Tests for simple effects of repetition carried out separately for attended or unattended objects revealed significant BOLD repetition suppression for attended objects $[F(1,12)=10.67, P=0.007]$ but not for unattended objects. If anything, there was a marginal tendency for the opposite pattern of BOLD repetition increases with unattended objects $[F(1,12)=4.84, P=0.048]$. We shall not base any strong conclusions on thatoutcome, which requires further replication. For now, the important point is that the results clearly show no evidence for BOLD repetition

\footnotetext{
${ }^{2}$ The view $\times$ attention interaction testing for larger differences in priming between attended and unattended original than mirror images yielded nothing except an activation at $P<0.001$, uncorrected, in the left intraparietal sulcus, but this did not survive correction for multiple comparisons across the volume of interest, and in any case fell outside the ventral visual areas which our hypotheses concerned. The reverse interaction contrast produced no effects at the same threshold.

${ }^{3}$ Note that the vast majority of subjects all showed positive behavioral priming from attended objects, but no significant behavioral priming (confidence intervals overlap with zero) from unattended objects. Analogously, the majority of subjects showed fMRI repetition-suppression effects from attended objects, but not from unattended objects.
}
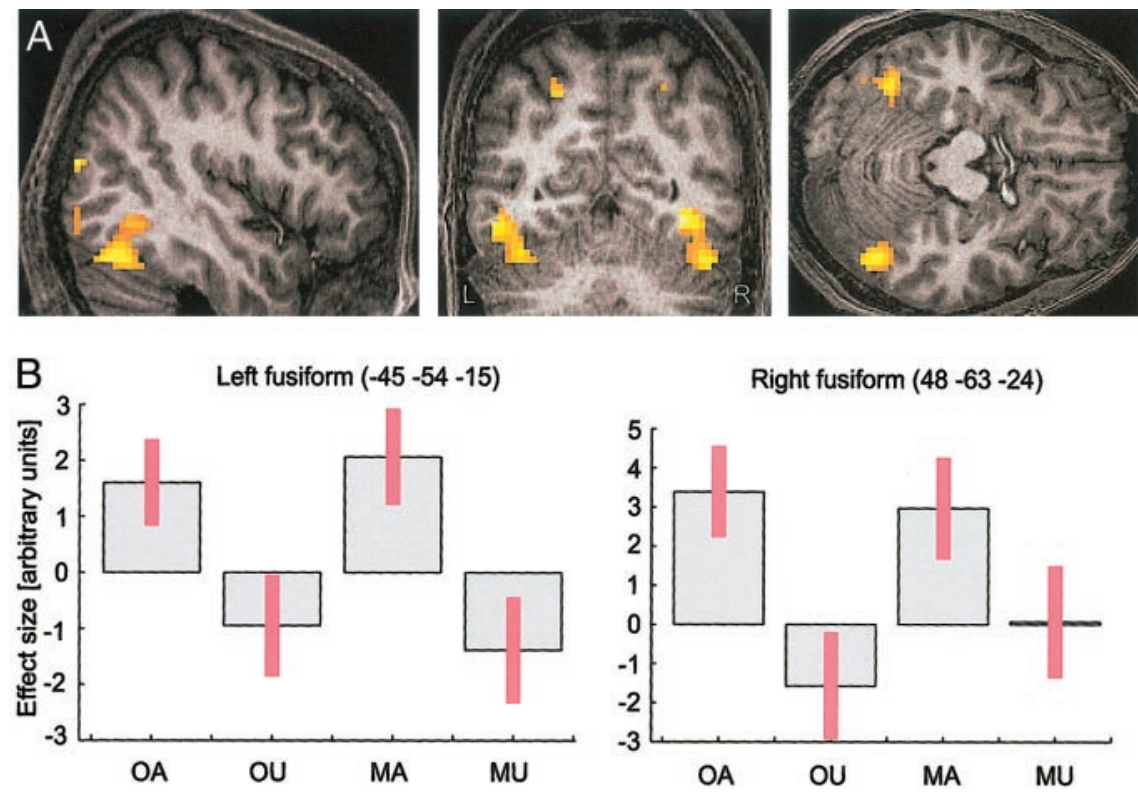

FIG. 3. Imaging findings from group analyses. A: shown are regions expressing larger repetition decreases for priming from attended than from unattended images collapsed across view. After subtraction from the appropriate unprimed baseline conditions. For display purposes, findings were color-coded at $P<0.001$, uncorrected (masked inclusively by objects $>$ phaserandomized noise at $P<0.001$ ) and superimposed on the normalized structural scan of one participating subject. $B$ : plots of responses (contrasts of parameter estimates) for the 4 priming conditions from the maxima in left and right lateral occipital and fusiform cortices (OA, priming from original image attended; OU, priming from original image unattended; MA, priming from mirror image attended; $\mathrm{MU}$, priming from mirror image unattended). Effects are displayed after subtraction from the unprimed baseline conditions; positive values thus denote repetition decreases. Error bars represent residual error from a one-way ANOVA on the 4 contrasts. 
TABLE 3. Factorial analysis (attended/unattended prime $\times$ original/mirror image) of the imaging data after subtraction from the respective baselines

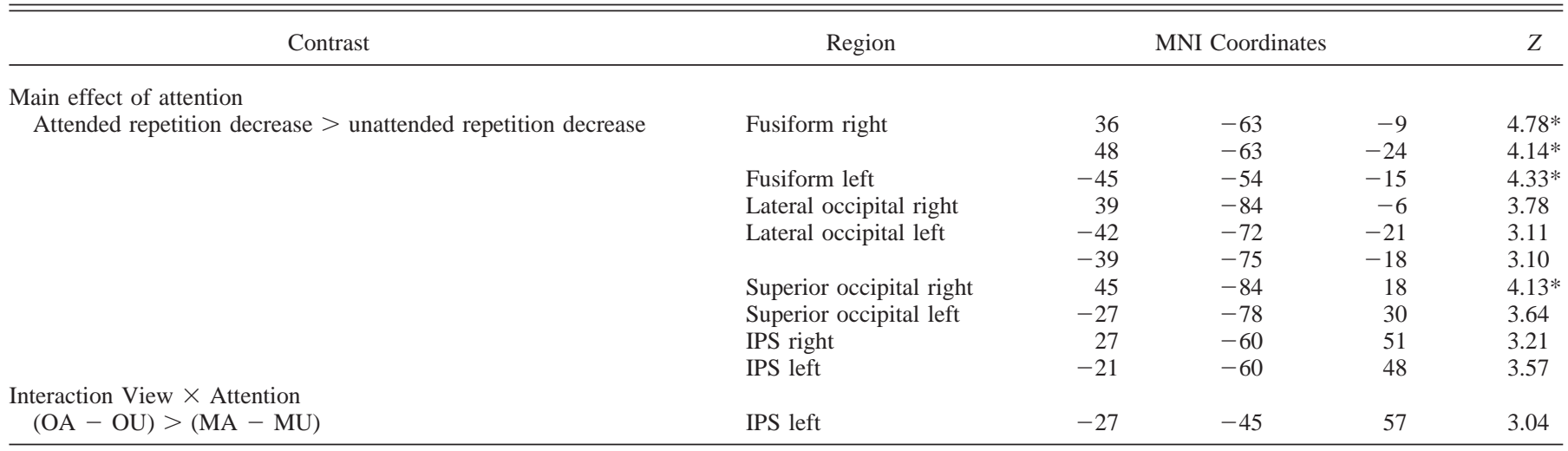

Regions that survived correction for multiple comparisons at voxel level for the volume of object-responsive areas, at $P<0.05$, are indicated with $*$ Other

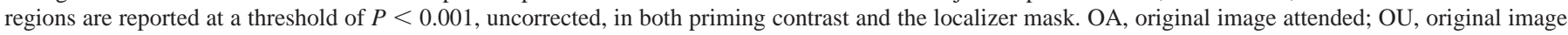
unattended; MA, mirror image attended; MU, mirror image unattended.

suppression from unattended objects, given that any residual tendency was actually in the opposite direction.

To summarize, attended objects produced reliable repetition effects in BOLD for both the same image and for repetition across a mirror transform, in lateral occipital and fusiform regions bilaterally. In striking contrast, no such repetition decreases were observed from unattended objects, demonstrating the dependency of these effects on attention, as confirmed by the main effect of our attentional factor.

\section{I S C U S S I O N}

The degree to which priming-related repetition decreases in BOLD depend on attention has received surprisingly little research in previous imaging studies (although see Murray and Wojciulik 2004). Some studies have found that behavioral priming accompanied by fMRI repetition suppression can occur with masked priming, and thus without explicit awareness of repetition (e.g., Dehaene et al. 2001). However, that situation is quite different from the spatial attention manipulation used here, where 2 objects were presented concurrently to "compete" for attentional resources. In the present situation, we demonstrated for the first time that BOLD repetition decreases for objects can be entirely dependent on spatial attention to the prime object.

One previously published study (Bentley et al. 2003) did assess fMRI repetition effects as a function of spatial attention. It reported that some repetition decreases in ventral occipitotemporal cortex may occur for unattended as well as attended faces. Faces rather than objects were used, and the apparent contrast with our finding may also relate to a weaker attentional manipulation in that study (e.g., longer presentation duration, less spatial separation) that might conceivably allow attentional resources to be deployed even to task-irrelevant stimuli. That study compared only second with first presentation of a given face, without including the appropriate baseline of unprimed items for unconfounding repetition, time, and order effects as here.

Another recent fMRI study (Murray and Wojciulik 2004) investigated adaptation in LO while directing attention either to schematic "objects" or to color changes at fixation. The effect of changes in object orientation on BOLD repetition suppres- sion was reduced when attending to the fixated colors, but repetition decreases were not completely abolished. The difference between the "unattended" results for that study and ours might relate to the strength of the attentional manipulations (Lavie 1995), to the stimuli used, or to the involvement of spatially cued attention (here) versus object-based attention; these are now issues for future work.

Our own behavioral and fMRI repetition effects clearly both depended strongly on spatial attention, but not on view (original or mirror image). This contrasts with the proposal by Stankiewicz et al. (1998) that same-view priming should be found without attention, whereas only priming across mirrorimage views should not. Our experiment differs from the purely behavioral work of Stankiewicz et al. in the format of the objects used (shaded grayscale instead of line drawings), and in the task used (size judgment instead of naming). Task factors can sometimes influence the outcome of behavioral priming, for instance with respect to the degree of image specificity (Bruce et al. 2000), although this does not undermine our demonstration that BOLD repetition-suppression effects can depend strongly on spatial attention.

The present fMRI repetition effects from attended primes generalized across view (for original and mirror images) in fusiform and lateral occipital cortex bilaterally (see Fig. 2), and did not interact with laterality in the ROI analysis. This differs from the proposal by Vuilleumier et al. (2002) that fMRI repetition effects may generalize across view changes more in the left than in the right hemispheres. However, these authors used more dissimilar and heterogeneous views (involving both plane and depth rotations), unlike the strict mirror imaging used here. Some models of object recognition that do not incorporate invariance to depth rotation can nevertheless generalize across mirror reversal (e.g., Riesenhuber and Poggio 1999). Moreover, invariance to mirror reversal in shape processing has been demonstrated in single-cell recordings in macaque inferotemporal cortex (Baylis and Driver 2001).

In conclusion, our results demonstrate that whereas repetition suppression for objects in ventral visual cortex generalizes across mirror reversal of the image, it is strongly dependent on spatial attention to the related prime, thus indicating that it requires attentional selection of relevant information in the 
A
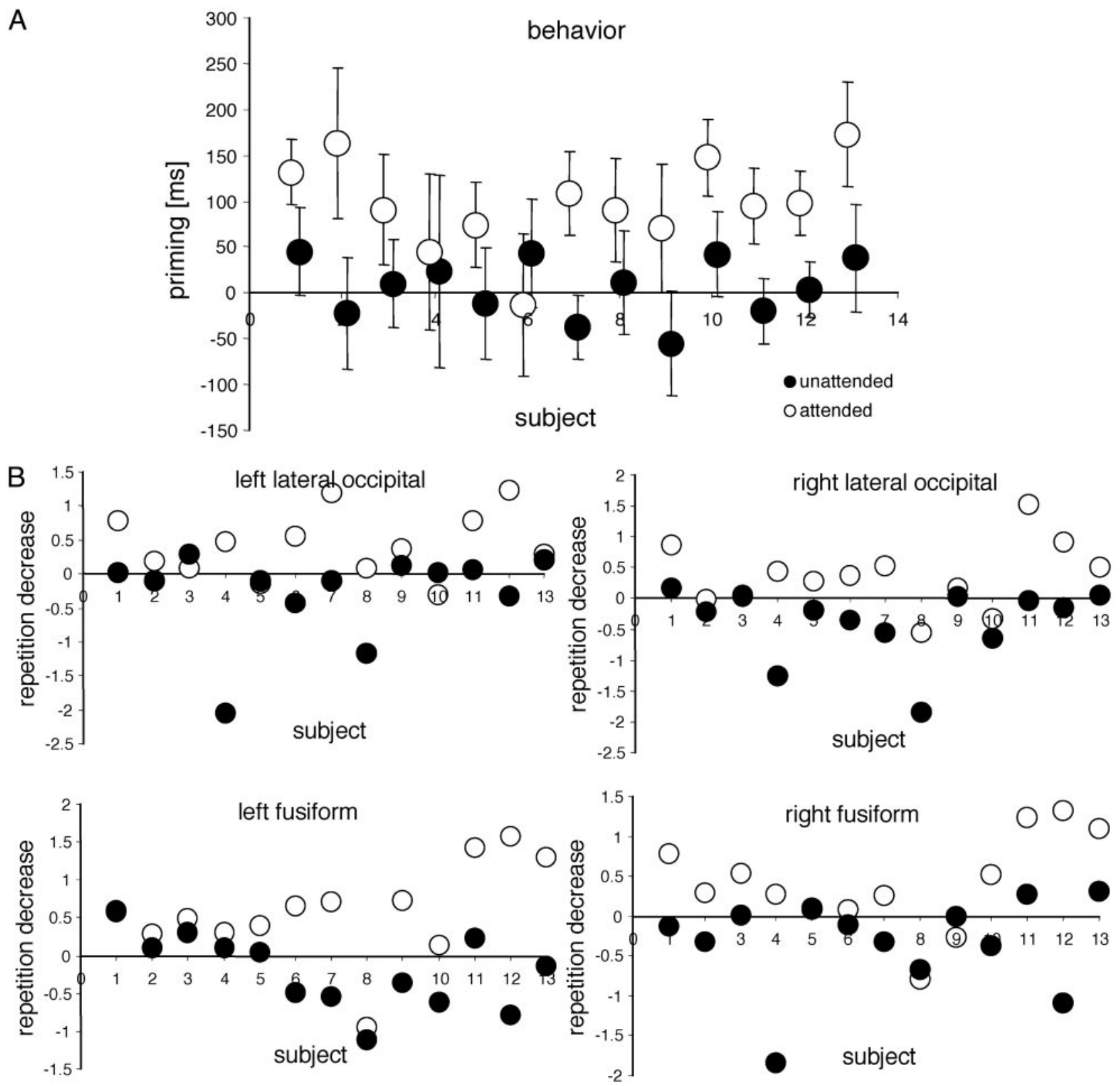

FIG. 4. Plots of individual behavioral and imaging repetition-related effects for the 13 subjects. $A$ : reaction time (RT) priming effects (unprimed - primed conditions, collapsed across view) for attended and unattended objects. Error bars indicate $95 \%$ confidence intervals. Note that the vast majority of subjects show reliable positive priming from attended objects (confidence intervals above zero), but no reliable priming from unattended objects (confidence intervals overlap with zero). B: individual contrasts for parameter estimates (unprimed - primed conditions; positive values thus denote repetition decreases) extracted from regions of interest of $10-\mathrm{mm}$ radius centered on individual lateral occipital and fusiform response maxima as determined by the separate object localizer scan.

visual input and is not an as automatic and intrinsic property of cortical cells (cf. Wiggs and Martin 1998), as often assumed.

\section{G R A N T S}

This study was supported by Programme grants from the Wellcome Trust to J. Driver and R. J. Dolan. R.N.A. Henson is supported by a Wellcome Trust Fellowship.

\section{REFERENCES}

Baylis GC and Driver J. Shape-coding in IT cells generalizes over contrast and mirror reversal, but not figure-ground reversal. Nat Neurosci 4: 937942,2001
Bentley P, Vuilleumier P, Thiel CM, Driver J, and Dolan RJ. Effects of attention and emotion on repetition priming and their modulation by cholinergic enhancement. J Neurophysiol 90: 1171-1181, 2003.

Biederman I and Kalocsai P. Neurocomputational bases of object and face recognition. Philos Trans R Soc Lond B Biol Sci 352: 1203-1219, 1997.

Bruce V, Carson D, Burton AM, and Ellis AW. Perceptual priming is not a necessary consequence of semantic classification of pictures. $Q J$ Exp Psychol A 53: 289-323, 2000.

Bulthoff HH, Edelman SY, and Tarr MJ. How are three-dimensional objects represented in the brain? Cereb Cortex 5: 247-260, 1995.

Crabb BT and Dark VJ. Perceptual implicit memory requires attentional encoding. Mem Cognit 27: 267-275, 1999. 
Dehaene S, Naccache L, Cohen L, Bihan DL, Mangin JF, Poline JB, and Riviere D. Cerebral mechanisms of word masking and unconscious repetition priming. Nat Neurosci 4: 752-758, 2001.

DeSchepper B and Treisman A. Visual memory for novel shapes: implicit coding without attention. J Exp Psychol Learn Mem Cogn 22: 27-47, 1996.

Grill-Spector K, Kushnir T, Edelman S, Avidan G, Itzchak Y, and Malach R. Differential processing of objects under various viewing conditions in the human lateral occipital complex. Neuron 24: 187-203, 1999.

Henson RN. Neuroimaging studies of priming. Prog Neurobiol 70: 53-81, 2003.

Henson RN and Rugg MD. Neural response suppression, haemodynamic repetition effects, and behavioural priming. Neuropsychologia 41: 263-270, 2003.

James TW, Humphrey GK, Gati JS, Menon RS, and Goodale MA. Differential effects of viewpoint on object-driven activation in dorsal and ventral streams. Neuron 35: 793-801, 2002.

Lavie N. Perceptual load as a necessary condition for selective attention. $J$ Exp Psychol Hum Percept Perform 21: 451-468, 1995.

Murray SO and Wojciulik E. Attention increases neural selectivity in the human lateral occipital complex. Nat Neurosci 7: 70-74, 2004.
Naccache $\mathbf{L}$ and Dehaene S. The priming method: imaging unconscious repetition priming reveals an abstract representation of number in the parietal lobes. Cereb Cortex 11: 966-974, 2001.

Riesenhuber $\mathbf{M}$ and Poggio T. Hierarchical models of object recognition in cortex. Nat Neurosci 2: 1019-1025, 1999.

Stankiewicz BJ, Hummel JE, and Cooper EE. The role of attention in priming for left-right reflections of object images: evidence for a dual representation of object shape. J Exp Psychol Hum Percept Perform 24: 732-744, 1998.

Tipper SP. The negative priming effect: inhibitory priming by ignored objects. Q J Exp Psychol A 37: 571-590, 1985.

Tipper SP and Driver J. Negative priming between pictures and words in a selective attention task: evidence for semantic processing of ignored stimuli. Mem Cognit 16: 64-70, 1988.

Vuilleumier P, Henson RN, Driver J, and Dolan RJ. Multiple levels of visual object constancy revealed by event-related fMRI of repetition priming. Nat Neurosci 5: 491-499, 2002.

Wiggs CL and Martin A. Properties and mechanisms of perceptual priming. Curr Opin Neurobiol 8: 227-233, 1998. 